\title{
Three-body problem with velocity-dependent optical potentials: a case of $(d, p)$ reactions
}

\author{
N. K. Timofeyuk \\ Department of Physics, Faculty of Engeneering and Physical Sciences, \\ Univesity of Surrey, Guildford, GU2 7XH, UK \\ E-mail: N.Timofeyuk@surrey.ac.uk
}

\begin{abstract}
The change in mass of a nucleon, arising from its interactions with other nucleons inside the target, results in velocity-dependent terms in the Schrödinger equation that describes nucleon scattering. It has recently been suggested in a number of publications that introducing and fitting velocity-dependent terms improves the quality of the description of nucleon scattering data for various nuclei. The present paper discusses velocity-dependent optical potentials in a context of a three-body problem used to account for deuteron breakup in the entrance channel of $(d, p)$ reactions. Such potentials form a particular class of nonlocal optical potentials which are a popular object of modern studies. It is shown here that because of a particular structure of the velocity-dependent terms the three-body problem can be formulated in two different ways. Solving this problem within an adiabatic approximation results in a significant difference between the two approaches caused by contributions from the high $n-p$ momenta in deuteron in one of them. Solving the three-body problem beyond the adiabatic approximation may remove such contributions, which is indirectly confirmed by replacing the adiabatic approximation by the folding Watanabe model where such contributions are suppressed. Discussion of numerical results is carried out for the ${ }^{40} \mathrm{Ca}(d, p){ }^{41} \mathrm{Ca}$ reaction where experimental data both on elastic scattering in entrance and exit channels and on nucleon transfer are available.
\end{abstract}


Three-body problem with velocity-dependent optical potentials: a case of $(d, p)$ reactions 2

\section{Introduction}

It has recently been pointed out that the introduction of velocity-dependent optical potentials improves the quality of the description of proton and neutron elastic scattering [1, 2, 3, 4, 5]. The origin of the velocity-dependence in these papers is attributed to a position-dependent effective mass of the nucleon moving in a nuclear medium, representing another source of nonlocality. Refs. [1, 3] cite a number of papers discussing the position-dependence of effective mass and point to a wider range of applications for velocity-dependent potentials such as in pion-nucleon, nucleon-nucleon and electronatom scattering as well as in describing the dynamics of electrons in semiconductors and quantum dots. For velocity-dependent effects in nuclear matter see [6].

On the other hand, it is also known that a nonlocal two-body problem is equivalent to one with a potential that contains an infinite sum of powers of kinetic energy operators arising from the Taylor series expansion of an exponent that contains the nucleon kinetic energy operator [7]. Thus, from a formal point of view, the velocity dependence used in Refs [1, 2, 3, 4, 5] is just a particular case of a more general nonlocal problem, truncated to retain linear terms only.

The present paper discusses the velocity-dependence of optical potentials in the context of a three-body $A+n+p$ problem and inquires how they perform when used to describe one nucleon transfer in $(d, p)$ reactions which are an important tool of spectroscopic studies of atomic nuclei. In recent years discussions of the role of nonlocality in $(d, p)$ studies became very popular $[8,49,10,11,12,13,14,15,16$, 17. 18, 19]. They were triggered by three-body Faddeev calculations in [8] showing an improvement of the differential $(d, p)$ cross sections description when nonlocality is introduced. A few years later, methods to treat deuteron breakup within an adiabatic approximation were developed [9, 10, 13, 16]. However, the adiabatic approximation was shown to induce strong sensitivity to the high $n$ - $p$ momenta in the incoming deuteron [15]. The sensitivity goes away when the $A+n+p$ problem is solved beyond the adiabatic approximation, which was observed both in a leading-order continuum discretized couped channel (CDCC) [17] and exact Fadeev [18] calculations. Extending the CDCC beyond the leading order is a non-trivial task while its development for nonlocal potentials of the velocity-dependent type introduced in [1] could be a simpler task. The possibility of future CDCC applications for this class of potentials partially motivates the present work.

The paper considers $(d, p)$ reactions within the Johnson-Tandy adiabatic distorted wave approximation (ADWA) [20]. It starts by describing the two-body problem with velocity-dependent potentials and their local-equivalents in section 2 . Then section 3 discusses the three-body Schrödinger equation. It is shown that velocitydependent forces could be used in two different ways determined by their two equivalent representations in the two-body channel. Adiabatic deuteron potentials are constructed in section 4 for both these models and numerical results are reported for the case of ${ }^{40} \mathrm{Ca}(d, p){ }^{41} \mathrm{Ca}$ reaction in section 5 . Conclusions are given in section 6 . 
Three-body problem with velocity-dependent optical potentials: a case of $(d, p)$ reactions3

\section{Velocity-dependent optical potentials in a two-body problem}

The optical potential parameterization in [1] was based on the fact that the change in mass of the nucleon, described by an isotropic function $\rho(r)$ and arising from its interactions with other nucleons inside the target, results in a velocity-dependence in the Schrödinger equation that has the simplest from given by

$$
\left(T+V(r)+\frac{\hbar^{2}}{2 \mu}\left[\rho(r) \nabla^{2}+\nabla \rho(r) \cdot \nabla\right]-E\right) \Psi=0,
$$

where $V(r)$ is the local optical potential that can include spin-orbit interaction. Here and everywhere below $\mu$ denotes the reduced mass of the $N+A$ system. Dividing Eq. (11) by $1-\rho(r)$ transforms it to an equivalent equation

$$
(T+\tilde{U}(r)+\nabla F(r) \cdot \nabla-E) \Psi=0,
$$

where the new potential $\tilde{U}$ and the velocity-dependent force $F$ are given by equations

$$
\begin{aligned}
& \tilde{U}(r)=\frac{V(r)-E \rho(r)}{1-\rho(r)}, \\
& F(r)=-\frac{\hbar^{2}}{2 \mu} \ln (1-\rho(r)) .
\end{aligned}
$$

Eq. (2) can be reduced to a standard form by introducing the Perey factor $P(r)$,

$$
\Psi(\boldsymbol{r})=P(\boldsymbol{r}) \varphi(\boldsymbol{r})
$$

and demanding that the equation describing $\varphi$ should not contain first derivatives. Then $P(\boldsymbol{r})$ satisfies the first-order differential equation

$$
\frac{\nabla P}{P}=\frac{\mu}{\hbar^{2}} \nabla F
$$

with the condition of $P \rightarrow 1$ when $r \rightarrow \infty$, while $\varphi(\boldsymbol{r})$ satisfies the local Schrödinger equation

$$
\left(T+\tilde{U}^{\mathrm{eff}}(r)-E\right) \varphi(\boldsymbol{r})=0,
$$

where $\tilde{U}^{\text {eff }}=\tilde{U}+\Delta U$ and

$$
\begin{aligned}
\Delta U & =-\frac{\hbar^{2}}{2 \mu} \frac{\nabla^{2} P}{P}+\frac{\nabla F \cdot \nabla P}{P} \\
& =-\frac{1}{2} \nabla^{2} F+\frac{1}{2} \frac{\mu}{\hbar^{2}}(\nabla F)^{2} .
\end{aligned}
$$

As discussed in [3], for a particular choice of $F$, given by Eq. (4),

$$
P(r)=\frac{1}{\sqrt{1-\rho(r)}} .
$$

This gives the following correction to the local-equivalent potential:

$$
\Delta U(r)=-\frac{h^{2}}{2 \mu}\left[\frac{1}{4}\left(\frac{\rho^{\prime}(r)}{1-\rho(r)}\right)^{2}+\frac{\rho^{\prime \prime}(r)}{2(1-\rho(r))}\right] .
$$


Three-body problem with velocity-dependent optical potentials: a case of $(d, p)$ reactions4

The above equations are written for neutron scattering. For protons, the Coulomb interaction $V_{c}$ should be added to Schrödinger equations (1), (2) and (17) while $\tilde{U}$ is now given by

$$
\tilde{U}(r)=\frac{V(r)-\left(E-V_{c}(r)\right) \rho(r)}{1-\rho(r)} .
$$

It should be noted that these transformations have been discussed in [1, 3] and the references thererin as well as in [6] and [21].

\section{Three-body problem with velocity-dependent nucleon optical potentials}

Let us consider a three-body $A+n+p$ problem with velocity-dependent $n-A$ and $p-A$ optical potentials. According to the previous section, the two-body nucleon scattering problem can be described in two exactly equivalent ways using the Schrödinger equation that contains either first derivatives only or both the first and the second derivatives. Below both cases will be considered in the context of the three-body problem.

\subsection{Case I: first derivatives only}

This case is based on the two-body interactions $\tilde{U}_{n A}$ and $\tilde{U}_{p A}$ given by model (2). The corresponding Schrödinger equation for the three-body wave function $\Psi(\boldsymbol{R}, \boldsymbol{r})$ reads

$$
\begin{aligned}
\left(T_{3}+V_{n p}(\boldsymbol{r})\right. & +\tilde{U}_{n A}\left(\boldsymbol{r}_{n}\right)+\nabla_{n} F_{n}\left(\boldsymbol{r}_{n}\right) \cdot \nabla_{n}+V_{p A}^{c}\left(r_{p}\right) \\
& \left.+\tilde{U}_{p A}\left(\boldsymbol{r}_{p}\right)+\nabla_{p} F_{p}\left(\boldsymbol{r}_{p}\right) \cdot \nabla_{p}-E\right) \Psi(\boldsymbol{R}, \boldsymbol{r})=0,
\end{aligned}
$$

where $\boldsymbol{r}_{n}$ and $\boldsymbol{r}_{p}$ are the coordinate-vectors of neutron and proton with respect to the target $A$, while $\boldsymbol{r}=\boldsymbol{r}_{n}-\boldsymbol{r}_{p}$ and $\boldsymbol{R}=\left(\boldsymbol{r}_{n}+\boldsymbol{r}_{p}\right) / 2$. Also, the $\nabla_{n}$ and $\nabla_{p}$ denote gradients with respect to variables $\boldsymbol{r}_{n}$ and $\boldsymbol{r}_{p}$ respectively. They are related to the gradients $\nabla_{R}$ and $\nabla_{r}$ in coordinates $\boldsymbol{R}$ and $\boldsymbol{r}$, respectively, as

$$
\begin{aligned}
& \nabla_{n}=\frac{1}{2} \nabla_{R}+\nabla_{r}, \\
& \nabla_{p}=\frac{1}{2} \nabla_{R}-\nabla_{r} .
\end{aligned}
$$

It is possible to reduce equation (12) to a form that does not contain first derivatives of variable $\boldsymbol{R}$. This is convenient for expanding the wave function $\Psi(\boldsymbol{R}, \boldsymbol{r})$ over some basis functions of variable $\boldsymbol{r}$. We will look for a solution for $\Psi(\boldsymbol{R}, \boldsymbol{r})$ in the form

$$
\Psi(\boldsymbol{R}, \boldsymbol{r})=P_{n}\left(\boldsymbol{r}_{n}\right) P_{p}\left(\boldsymbol{r}_{p}\right) \varphi(\boldsymbol{R}, \boldsymbol{r})
$$

requiring $P_{i}\left(r_{i}\right) \rightarrow 1$ for $r_{i} \rightarrow \infty$. Substituting it into Eq. (12) and demanding that there are no terms containing first derivatives over $R$ we obtain

$$
-\frac{\hbar^{2}}{\mu_{d A}} \nabla_{R}\left[P_{n} P_{p}\right]+\frac{1}{2} P_{n} P_{p}\left(\nabla_{n} F_{n}+\nabla_{p} F_{p}\right)=0,
$$

where $\mu_{d A}=m_{A}\left(m_{n}+m_{p}\right) /\left(m_{A}+m_{n}+m_{p}\right)$ and $m_{i}$ is the mass of nucleus $i$. Since $\nabla_{R}=\nabla_{n}+\nabla_{p}$, Eq. (15) after division by $P_{n} P_{p}$ becomes equivalent to

$$
-\frac{\hbar^{2}}{\mu_{d A}}\left(\frac{\nabla_{n} P_{n}}{P_{n}}+\frac{\nabla_{p} P_{p}}{P_{p}}\right)+\frac{1}{2}\left(\nabla_{n} F_{n}+\nabla_{p} F_{p}\right)=0,
$$


Three-body problem with velocity-dependent optical potentials: a case of $(d, p)$ reactions5

which can only be achieved when

$$
\frac{\nabla_{N} P_{N}}{P_{N}}=\frac{\mu_{d A}}{2 \hbar^{2}} \nabla_{N} F_{N}
$$

both for neutrons $(N=n)$ and protons $(N=p)$. Eq. (17) is almost identical to (6) except for the reduced mass, which is different by the factor of $\left(m_{A}+1\right) /\left(m_{A}+2\right)$. With this choice of $P_{n}$ and $P_{p}$ the wave function $\varphi(\boldsymbol{R}, \boldsymbol{r})$ satisfies the Schrödinger equation with local nucleon optical potentials, consisting of the original local $\tilde{U}_{N A}$ potentials with some Perey-factor-based corrections, and with additional contributions that could be considered as a three-body force because they depend on the positions of both the neutron and the proton at the same time:

$$
\begin{aligned}
{\left[T_{3}\right.} & +V_{n p}(\boldsymbol{r})+\tilde{U}_{n A}\left(\boldsymbol{r}_{n}\right)+\tilde{U}_{p A}\left(\boldsymbol{r}_{p}\right)+V_{p A}^{c}\left(r_{p}\right)+\left[\frac{\nabla_{n} P_{n} \cdot \nabla_{n} F_{n}}{P_{n}}+\frac{\nabla_{p} P_{p} \cdot \nabla_{p} F_{p}}{P_{p}}\right] \\
& +\left(1-\frac{\mu_{d A}}{4 \mu_{n p}}\right)\left(\nabla_{n} F_{n}-\nabla_{p} F_{p}\right) \cdot \nabla_{r}-\left(\frac{\hbar^{2}}{\mu_{d A}}-\frac{\hbar^{2}}{4 \mu_{n p}}\right) \frac{\nabla_{n} P_{n} \cdot \nabla_{p} P_{p}}{P_{n} P_{p}} \\
& \left.-\left(\frac{\hbar^{2}}{2 \mu_{d A}}+\frac{\hbar^{2}}{8 \mu_{n p}}\right) \frac{\nabla_{n}^{2} P_{n}}{P_{n}}-\left(\frac{\hbar^{2}}{2 \mu_{d A}}+\frac{\hbar^{2}}{8 \mu_{n p}}\right) \frac{\nabla_{p}^{2} P_{p}}{P_{p}}-E\right] \varphi(\boldsymbol{R}, \boldsymbol{r})=0 .
\end{aligned}
$$

One of these contributions has $n-p$ velocity-dependence that comes through $\nabla_{r}$. If one assumes all reduced masses are determined by nuclei mass numbers only then

$$
\begin{aligned}
& \frac{1}{\mu_{d A}}+\frac{1}{4 \mu_{n p}}=\frac{1}{\mu}, \\
& \frac{1}{\mu_{d A}}-\frac{1}{4 \mu_{n p}}=\frac{1}{M_{A}},
\end{aligned}
$$

where $\mu$ is the reduced mass of the $A+1$ system, the same one that features in the two-body problem, and $M_{A}$ is the mass of the target $A$. In this case the Schrödinger equation reduces to

$$
\begin{aligned}
& {\left[T_{3}-E+V_{n p}(\boldsymbol{r})+\tilde{U}_{n A}^{\mathrm{eff}}\left(\boldsymbol{r}_{n}\right)+\tilde{U}_{p A}^{\mathrm{eff}}\left(\boldsymbol{r}_{p}\right)+V_{p A}^{c}\left(r_{p}\right)\right.} \\
& \left.\quad+\frac{2}{A+2}\left(\nabla_{n} F_{n}-\nabla_{p} F_{p}\right) \cdot \nabla_{r}-\frac{\mu_{d A}^{2}}{4 \hbar^{2} M_{A}} \nabla_{n} F_{n} \cdot \nabla_{p} F_{p}\right] \varphi(\boldsymbol{R}, \boldsymbol{r})=0
\end{aligned}
$$

where the effective $N-A$ potentials are given by

$$
\tilde{U}_{N A}^{\mathrm{eff}}=\tilde{U}_{N A}-\frac{1}{2} \frac{A+1}{A+2} \nabla_{N}^{2} F_{N}+\left(1-\frac{1}{2} \frac{A+1}{A+2}\right) \frac{\mu_{d A}}{2 \hbar^{2}}\left(\nabla_{N} F_{N}\right)^{2} .
$$

They differ from two-body effective potentials of section 2 by additional mass-dependent factors that vanish when $A \rightarrow \infty$.

\subsection{Case II: first and second derivatives}

This case is based on the original model (1) with local interaction $V_{N A}(r)$. The corresponding Schrödinger equation for the three-body wave function $\Psi(\boldsymbol{R}, \boldsymbol{r})$ reads

$$
\left(T_{3}+V_{n p}(\boldsymbol{r})+V_{n A}\left(\boldsymbol{r}_{n}\right)+\frac{\hbar^{2}}{2 \mu}\left(\nabla_{n} \rho_{n}\left(r_{n}\right) \cdot \nabla_{n}+\rho_{n}\left(r_{n}\right) \nabla_{n}^{2}\right)+V_{p A}^{c}\left(r_{p}\right)\right.
$$


Three-body problem with velocity-dependent optical potentials: a case of $(d, p)$ reactions6

$$
\left.+V_{p A}\left(\boldsymbol{r}_{p}\right)+\frac{\hbar^{2}}{2 \mu}\left(\nabla_{p} \rho_{p}\left(r_{p}\right) \cdot \nabla_{p}+\rho_{p}\left(r_{p}\right) \nabla_{p}^{2}\right)-E\right) \Psi(\boldsymbol{R}, \boldsymbol{r})=0 .
$$

Because of the second-order derivatives $\nabla_{n}^{2}$ and $\nabla_{p}^{2}$, the introduction of the Perey factors from the previous subsection will result in an additional term $\left(\nabla_{R} \cdot \nabla_{r}\right)$ in the Schrödinger equation (20), which does not bring any advantages. Therefore, no preliminary modifications of Eq. (22) have been done.

\section{Adiabatic approximation for $(d, p)$ reactions with velocity-dependent potentials}

The $(d, p)$ transition amplitude contains the short-range interaction $V_{n p}[22$ and therefore the $(d, p)$ cross sections are determined by the wave function $\Psi(\boldsymbol{R}, \boldsymbol{r})$ at very small values of $r$. Usually, for local $V_{N A}$ potentials, $\Psi(\boldsymbol{R}, \boldsymbol{r})$ is expanded over the Weinberg state basis and only the first term of this expansion is retained thus assuming that $\Psi(\boldsymbol{R}, \boldsymbol{r}) \approx \chi(\boldsymbol{R}) \phi_{0}(\boldsymbol{r})$, where $\phi_{0}$ is the $s$-wave deuteron wave function, is a good approximation in the small $r$ region [20]. In this case, $\chi(\boldsymbol{R})$ satisfies the two-body Schrödinger equation with the Johnson-Tandy adiabatic potential

$$
U_{d A}^{J T}(\boldsymbol{R})=\int d \boldsymbol{r} \phi_{1}^{*}(\boldsymbol{r})\left[V_{n A}\left(\boldsymbol{R}+\frac{\boldsymbol{r}}{2}\right)+V_{p A}\left(\boldsymbol{R}-\frac{\boldsymbol{r}}{2}\right)\right] \phi_{0}(\boldsymbol{r}),
$$

where

$$
\phi_{1}(r)=\frac{\phi_{0}(r)}{\left\langle\phi_{0}\left|V_{n p}\right| \phi_{0}\right\rangle} .
$$

In this paper, the same adiabatic approximation with the $s$-wave deuteron is used. Including the $d$-wave does not change $U_{d A}^{J T}(\boldsymbol{R})$ if the $N-A$ potentials are local [16], however, it produces dramatic changes in the case of nonlocal potentials [15] which are an artefact of the adiabatic approximation [17, 18].

In the case I of the previous section, the adiabatic approximation could be introduced either before or after introduction of the Perey factors. Both cases will be considered here, labelled by $(a)$, which corresponds to introduction of adiabatic approximation after introduction of Perey factor,

$$
(a): \quad \varphi(\boldsymbol{R}, \boldsymbol{r})=\chi(\boldsymbol{R}) \phi_{0}(\boldsymbol{r}),
$$

or $(b)$, which corresponds to introduction of adiabatic approximation before the Perey factors are introduced:

$$
\text { (b) : } \quad \Psi(\boldsymbol{R}, \boldsymbol{r})=\chi(\boldsymbol{R}) \phi_{0}(\boldsymbol{r}) .
$$

As for the case II, the adiabatic approximation will be introduced from the very beginning using approximation (26). 
Three-body problem with velocity-dependent optical potentials: a case of $(d, p)$ reactions7

\subsection{Case Ia}

Using approximation (25) in Eq. (20) one obtains the following two-body Schrödinger equation for $\chi(\boldsymbol{R})$ :

$$
\left[T_{2}+\tilde{U}_{d A}^{(0)}(\boldsymbol{R})+\Delta U_{1}(\boldsymbol{R})+\Delta U_{2}(\boldsymbol{R})+V_{d A}^{c}(R)-E_{d}\right] \chi(\boldsymbol{R})=0
$$

where $T_{2}$ is the kinetic energy operator associated with variable $\boldsymbol{R}, E_{d}=E+\varepsilon$ is the centre-of-mass energy of the incoming deuteron ( $\varepsilon$ being the (negative) deuteron binding energy) and

$$
\tilde{U}_{d A}^{(0)}(\boldsymbol{R})=\int d \boldsymbol{r} \phi_{1}^{*}(\boldsymbol{r})\left[\tilde{U}_{n A}^{\mathrm{eff}}\left(\boldsymbol{R}+\frac{\boldsymbol{r}}{2}\right)+\tilde{U}_{p A}^{\mathrm{eff}}\left(\boldsymbol{R}-\frac{\boldsymbol{r}}{2}\right)\right] \phi_{0}(\boldsymbol{r})
$$

is analogous to the commonly used Johnson-Tandy potential (23). In this paper, a usual assumption is made that the Johnson-Tandy potential associated with the Coulomb $p-A$ potential results in the $d-A$ Coulomb potential without any polarization terms. Other terms in Eq. (27) are

$$
\begin{aligned}
& \Delta U_{1}(\boldsymbol{R})=\frac{2}{A+2} \int d \boldsymbol{r} \phi_{1}^{*}(\boldsymbol{r})\left(\nabla_{n} F_{n}-\nabla_{p} F_{p}\right) \cdot \nabla_{r} \phi_{0}(\boldsymbol{r}), \\
& \Delta U_{2}(\boldsymbol{R})=-\frac{\mu_{d A}^{2}}{4 \hbar^{2} M_{A}} \int d \boldsymbol{r} \phi_{1}^{*}(\boldsymbol{r})\left(\nabla_{n} F_{n} \cdot \nabla_{p} F_{p}\right) \phi_{0}(\boldsymbol{r}) .
\end{aligned}
$$

To estimate these additional terms, the $F_{n}$ and $F_{p}$ are assumed to be sphericallysymmetric. In this case

$$
\nabla_{i} F_{i}=\boldsymbol{e}_{i} \frac{\partial F_{i}\left(r_{i}\right)}{\partial r_{i}}=\boldsymbol{e}_{i} F_{i}^{\prime}
$$

where $\boldsymbol{e}_{i}$ is the unit vector in the direction of $\boldsymbol{r}_{i}$. Similarly,

$$
\nabla_{r} \phi_{0}(\boldsymbol{r})=\boldsymbol{e}_{r} \phi_{0}^{\prime}(r)
$$

(note that $\phi_{0}(r)$ includes $Y_{00}(\hat{\boldsymbol{r}})$ ). Using relations

$$
\begin{aligned}
& \left(\boldsymbol{e}_{n} \cdot \boldsymbol{e}_{r}\right)=\frac{1}{r_{n}}\left(R \nu+\frac{1}{2} r\right), \\
& \left(\boldsymbol{e}_{p} \cdot \boldsymbol{e}_{r}\right)=\frac{1}{r_{p}}\left(R \nu-\frac{1}{2} r\right), \\
& \left(\boldsymbol{e}_{n} \cdot \boldsymbol{e}_{p}\right)=\frac{1}{r_{n} r_{p}}\left(R^{2}-\frac{1}{4} r^{2}\right),
\end{aligned}
$$

where $\nu=\cos (\hat{\boldsymbol{R}, \boldsymbol{r}})$ one obtains

$$
\begin{aligned}
& \Delta U_{1}(R)=\frac{2}{A+2} \int d \boldsymbol{r} \phi_{1}(r)\left[\frac{r}{2}\left(\frac{F_{n}^{\prime}\left(r_{n}\right)}{r_{n}}+\frac{F_{p}^{\prime}\left(r_{p}\right)}{r_{p}}\right)\right. \\
&+\left.R \nu\left(\frac{F_{n}^{\prime}\left(r_{n}\right)}{r_{n}}-\frac{F_{p}^{\prime}\left(r_{p}\right)}{r_{p}}\right)\right] \phi_{0}^{\prime}(r), \\
& \Delta U_{2}(R)=-\frac{\mu_{d A}^{2}}{4 \hbar^{2} M_{A}} \int d \boldsymbol{r} \phi_{1}^{*}(\boldsymbol{r}) \frac{F_{n}^{\prime}\left(r_{n}\right) F^{\prime}\left(r_{p}\right)}{r_{n} r_{p}}\left(R^{2}-\frac{1}{4} r^{2}\right) \phi_{0}(\boldsymbol{r}) .
\end{aligned}
$$

in which $r_{n}=\sqrt{R^{2}+\nu r R+r^{2} / 4}$ and $r_{p}=\sqrt{R^{2}-\nu r R+r^{2} / 4}$. 
Three-body problem with velocity-dependent optical potentials: a case of $(d, p)$ reactions 8

\subsection{Case $\mathrm{Ib}$}

Making approximation (26) in eq. (12) one obtains for distorted wave $\chi(\boldsymbol{R})$ the equation

$$
\left[T_{2}+\tilde{U}_{d A}^{(0)}(\boldsymbol{R})+\boldsymbol{S}(\boldsymbol{R}) \cdot \nabla_{R}+D(\boldsymbol{R})+V_{d A}^{c}(R)-E_{d}\right] \chi(\boldsymbol{R})=0,
$$

where

$$
\tilde{U}_{d A}^{(0)}(\boldsymbol{R})=\int d \boldsymbol{r} \phi_{1}^{*}(\boldsymbol{r})\left[\tilde{U}_{n A}\left(\boldsymbol{R}+\frac{\boldsymbol{r}}{2}\right)+\tilde{U}_{p A}\left(\boldsymbol{R}-\frac{\boldsymbol{r}}{2}\right)\right] \phi_{0}(\boldsymbol{r}),
$$

is the Johnson-Tandy potential based on the original local-equivalent potentials $\tilde{U}_{N A}$, and

$$
\begin{aligned}
& \boldsymbol{S}(\boldsymbol{R})=\frac{1}{2} \int d \boldsymbol{r} \phi_{1}(\boldsymbol{r})\left[\nabla_{n} F_{n}+\nabla_{p} F_{p}\right] \phi_{0}(\boldsymbol{r}), \\
& D(\boldsymbol{R})=\frac{A+2}{2} \Delta U_{1}(\boldsymbol{R}) .
\end{aligned}
$$

It can be shown that for a spherically-symmetrical velocity-dependent force $F_{N}$ and for the $s$-wave deuteron the term $\boldsymbol{S}(\boldsymbol{R}) \cdot \nabla_{R}$ is equal to

$$
\boldsymbol{S}(\boldsymbol{R}) \cdot \nabla_{R}=S(R) \frac{\partial}{\partial R} .
$$

This can be obtained using Eqs. (31), (32) and relations $\boldsymbol{e}_{R} \cdot \nabla_{R}=\frac{\partial}{\partial R}$ and

$$
\int d \boldsymbol{r} F(|\boldsymbol{r}-\boldsymbol{R}|) G(r) \boldsymbol{e}_{r} \cdot \nabla_{R}=\int_{0}^{\infty} d r r^{2} G(r) \mathcal{F}_{1}(r, R) \frac{\partial}{\partial R}
$$

where $\mathcal{F}_{\lambda}(r, R)$ is the radial part of the multipole expansion of $F(\boldsymbol{r}, \boldsymbol{R})$,

$$
F(|\boldsymbol{r}-\boldsymbol{R}|)=\sum_{\lambda \mu} \mathcal{F}_{\lambda}(r, R) Y_{\lambda \mu}^{*}(\hat{\boldsymbol{r}}) Y_{\lambda \mu}(\hat{\boldsymbol{R}}) .
$$

$S(R)$ is given by

$$
S(R)=\int_{0}^{\infty} d r r^{2} \phi_{1}(r)\left[\frac{1}{2} \mathcal{F}_{0}(r, R)+\frac{1}{4} \mathcal{F}_{1}(r, R)\right] \phi_{0}(r)
$$

with

$$
\begin{aligned}
& \mathcal{F}_{0}(r, R)=2 \pi R \int_{-1}^{1} d \mu\left(\frac{F_{n}^{\prime}\left(r_{n}\right)}{r_{n}}+\frac{F_{p}^{\prime}\left(r_{p}\right)}{r_{p}}\right), \\
& \mathcal{F}_{1}(r, R)=2 \pi r \int_{-1}^{1} d \mu \mu\left(\frac{F_{n}^{\prime}\left(r_{n}\right)}{r_{n}}-\frac{F_{p}^{\prime}\left(r_{p}\right)}{r_{p}}\right) .
\end{aligned}
$$

The Perey factor $P(R)$ can be now introduced in the usual way, $\chi(R)=P(R) \varphi(R)$. $P(R)$ satisfies the equation

$$
\frac{P^{\prime}(R)}{P(R)}=\frac{\mu_{d A}}{\hbar^{2}} S(R)
$$

while $\varphi(R)$ is the solution of the ordinary Schrödinger equation

$$
\left(T_{2}+U_{d A}^{\mathrm{eff}}(R)+V_{d A}^{c}(R)-E_{d}\right) \varphi(R)=0
$$

with the effective potential

$$
U^{\mathrm{eff}}(R)=\tilde{U}_{d A}^{(0)}(R)+V_{d A}^{c}(R)+\frac{1}{2} \frac{\mu_{d A}}{\hbar^{2}} S^{2}(R)-\frac{1}{2} S^{\prime}(R)+D(R) .
$$


Three-body problem with velocity-dependent optical potentials: a case of $(d, p)$ reactions9

\subsection{Case II}

One can show that applying approximation (26) in the three-body Schrödinger equation (22) results in a two-body differential equation similar to Eq. (38) that describes the case $\mathrm{Ib}$ but with an additional term that arises due to the second derivatives in the velocity-dependent force,

$$
\begin{aligned}
& \frac{\hbar^{2}}{2 \mu} \int d \boldsymbol{r} \phi_{1}^{*}(\boldsymbol{r})\left(\rho_{n} \nabla_{n}^{2}+\rho_{p} \nabla_{p}^{2}\right) \phi_{0}(\boldsymbol{r}) \\
& =\frac{\hbar^{2}}{2 \mu} \int d \boldsymbol{r} \phi_{1}^{*}(\boldsymbol{r})\left[\frac{1}{4}\left(\rho_{n}+\rho_{p}\right) \nabla_{R}^{2}+\left(\rho_{n}+\rho_{p}\right) \nabla_{r}^{2}+\left(\rho_{n}-\rho_{p}\right)\left(\nabla_{r} \cdot \nabla_{R}\right)\right] \phi_{0}(\boldsymbol{r}) .
\end{aligned}
$$

This two-body equation reads

$$
\begin{aligned}
\left(-\frac{\hbar^{2}}{2 \mu_{d A}}(1-\mathcal{R}(R)) \nabla_{R}^{2}\right. & +V_{d A}^{J T}(R)+V_{d A}^{c}(R)+B(R)+\tilde{D}(R) \\
& \left.+\left(S_{1}(R)+S_{2}(R)\right) \frac{\partial}{\partial R}-E_{d}\right) \chi(\boldsymbol{R})=0
\end{aligned}
$$

where $V_{d A}^{J T}(R)$ is the usual Johnson-Tandy potential, constructed from the original local potentials $V_{n}$ and $V_{p}$, and

$$
\begin{aligned}
& \mathcal{R}(R)=\frac{\mu_{d A}}{4 \mu} \int d \boldsymbol{r} \phi_{1}(\boldsymbol{r})\left(\rho_{n}+\rho_{p}\right) \phi_{0}(\boldsymbol{r}), \\
& B(R)=\frac{\hbar^{2}}{2 \mu} \int d \boldsymbol{r} \phi_{1}(\boldsymbol{r})\left(\rho_{n}+\rho_{p}\right) \nabla_{r}^{2} \phi_{0}(\boldsymbol{r}) .
\end{aligned}
$$

The $S_{1}(R)$ and $\tilde{D}(R)$ are equivalent to $S(R)$ and $D(R)$ from the previous subsection and they are given by Eqs. (40) and (41), respectively, by replacing $F_{N}^{\prime}$ by $\hbar^{2} /(2 \mu) \rho_{N}$. The $S_{2}(R)$ in (51) is

$$
S_{2}(R)=\int d \boldsymbol{r} \nu \phi_{1}(r)\left[\rho_{n}\left(r_{n}\right)-\rho_{p}\left(r_{p}\right) \phi_{0}^{\prime}(r) .\right.
$$

Again, as in sec. II, dividing (51) by $(1-\mathcal{R}(R))$ results in

$$
\left(T_{2}+\tilde{V}_{d A}(R)+\tilde{S}(R) \frac{\partial}{\partial R}+V_{d A}^{c}(R)-E_{d}\right) \chi(\boldsymbol{R})=0,
$$

where

$$
\begin{aligned}
& \tilde{V}_{d A}(R)=\frac{V_{d A}^{J T}(R)-\left[E_{d}-V_{d A}^{c}(R)\right] \mathcal{R}(R)+B(R)+\tilde{D}(R)}{1-\mathcal{R}(R)}, \\
& \tilde{S}(R)=\frac{S_{1}(R)+S_{2}(R)}{1-\mathcal{R}(R)} .
\end{aligned}
$$

As in the case Ib, the introduction of the Perey factor through $\chi(R)=P(R) \varphi(R)$ will help to get rid of the first derivatives over $R$. The $P(R)$ and $\varphi(R)$ are given by Eq. (48) and (49)-(50) in which $S(R)$ is substituted by $\tilde{S}(R)$. 
Three-body problem with velocity-dependent optical potentials: a case of $(d, p)$ reactions10

\section{Numerical calculations for ${ }^{40} \mathrm{Ca}(\mathrm{d}, \mathrm{p}){ }^{41} \mathrm{Ca}$ reaction}

In this sections the ${ }^{40} \mathrm{Ca}(\mathrm{d}, \mathrm{p}){ }^{41} \mathrm{Ca}$ cross sections are calculated for the deuteron incident laboratory energy of $20 \mathrm{MeV}$ in the zero-range approximation. Calculations are done with the help of the latest version of the code TWOFNR [23] which has an option to read distorted waves in, useful for modifying them externally by non-standard Perey factors.

The ADWA uses nucleon optical potentials taken at half the deuteron incident energy - the tradition that goes back to works [24, 20]. Experimental data on neutron and proton scattering at $10 \mathrm{MeV}$ and optical potentials are available. First of all, velocity-dependent optical potential from Ref. [2] was used in this paper. It is described by the function

$$
\rho(r)=\rho_{0} a_{\rho} \frac{d}{d r} \frac{1}{1+\exp \left[\left(r-r_{\rho} A^{1 / 3}\right) / a_{\rho}\right]}
$$

with $\rho_{0}=-1.9, r_{\rho}=1.63 \mathrm{fm}$ and $a_{\rho}=0.25 \mathrm{fm}$. It was found that the local equivalent potentials $\tilde{U}^{\text {eff }}$, constructed with these parameters, have a huge repulsive narrow peak (with the height of the order of $60 \mathrm{MeV}$ ) in the surface region, which is clearly unphysical (see Fig. 11). This peak originates due to a very narrow width of $\rho(r)$ determined by a small diffuseness parameter of $0.25 \mathrm{fm}$. The second derivative of this narrow function, that determines $\Delta U$ in eq. (10), is very large. The local equivalent potential $\tilde{U}^{\text {eff }}$ in the exit channel looks even more unphysical. Because of the presence of the volume term in $\rho(r)$ [4] at the energy of the exit proton channel, the depth of $\tilde{U}^{\text {eff }}$ is only about $10 \mathrm{MeV}$, which severely affects the $(d, p)$ cross sections. The unphysical behaviour of the nucleon local equivalent potentials, based on parameters of [2], translates into unphysical behaviour of deuteron adiabatic potentials and results in a huge differences between adiabatic deuteron potentials calculated in models Ia, Ib and II. For this reason, the potentials of Refs. [2, 4] were abandoned and a more conventional diffuseness was adopted for $\rho(r)$.

In all calculations below, the frequently used Becchetti-Greenlees optical potential
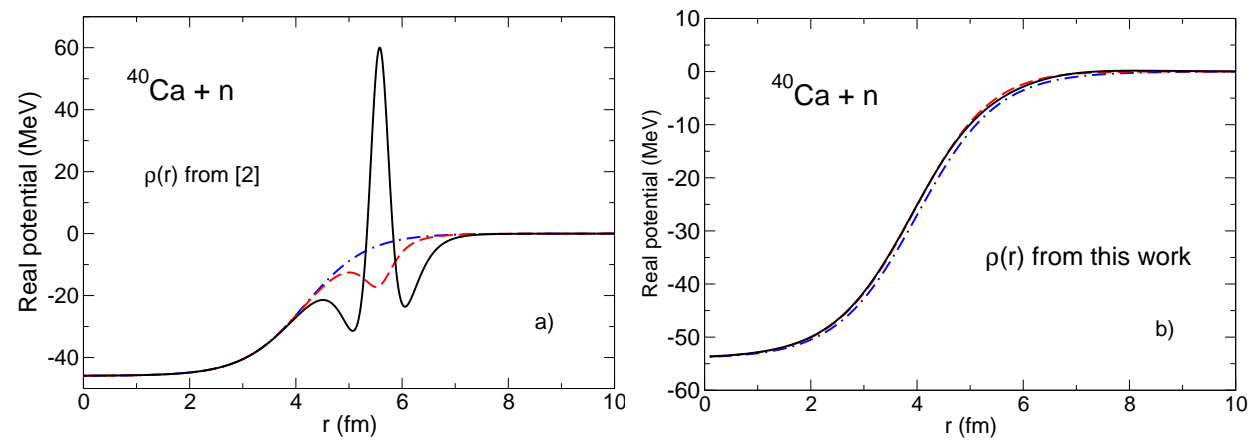

Figure 1. The real parts of the $n-{ }^{40} \mathrm{Ca}$ potentials $V(r)$ (dot-dash line), $\tilde{U}$ (dashed line) and $\tilde{U}^{\text {eff }}$ (solid line) calculated (a) for $11 \mathrm{MeV}$ neutrons using velocity-dependent potential from [2] and (b) for $10 \mathrm{MeV}$ neutrons using Becchetti-Greenlees potential and $\rho(r)$ fitted in this work. 
[25] was used, to which a velocity-dependent term was added with the parameters fitted to improve the description of the elastic scattering data from the ${ }^{40} \mathrm{Ca}$ target for neutrons at $10 \mathrm{MeV}$ [26] and for protons at 10 and $22 \mathrm{MeV}$ [27]. It was found that the choice of $\rho_{0}=0.4, r_{\rho}=1.63 \mathrm{fm}$ and $a_{\rho}=0.90 \mathrm{fm}$ somewhat improves the description of elastic scattering of neutrons and protons on ${ }^{40} \mathrm{Ca}$ at $10 \mathrm{MeV}$ (see Fig. 21). The same set of parameters improves description of the $22 \mathrm{MeV}$ data for $p+{ }^{40} \mathrm{Ca}$ at large angles while making it a bit worse for $60 \leq \theta \leq 120$ degrees. The general quality of the data fit is similar to the one with the original Becchetti-Greenless potential. These new values of $\rho_{0}, r_{\rho} \mathrm{fm}$ and $a_{\rho}$ are used below. It should be noted that a positive value of $\rho_{0}$ was essential to improvement of the data fit. Negative values deteriorated it significantly. It should also be mentioned that the surface form of $\rho(r)$, chosen in refs. [1, 2, 3], gives a surface-peaked effective nucleon mass $m^{*}(r) / m=1 /(1-\rho(r))$, equal to the square of the nucleon Perey factor. Detailed mean-field calculations of variable nucleon mass in finite nuclei suggest that $m^{*}(r) / m$ has a volume form, decreasing from 1 at large $r$ to 0.8 at $r=0$ [28]. In this paper, the effects of the surface form of $\rho(r)$ are investigated.

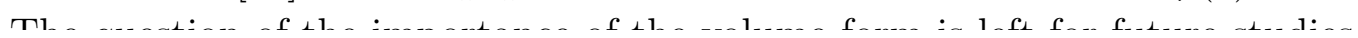
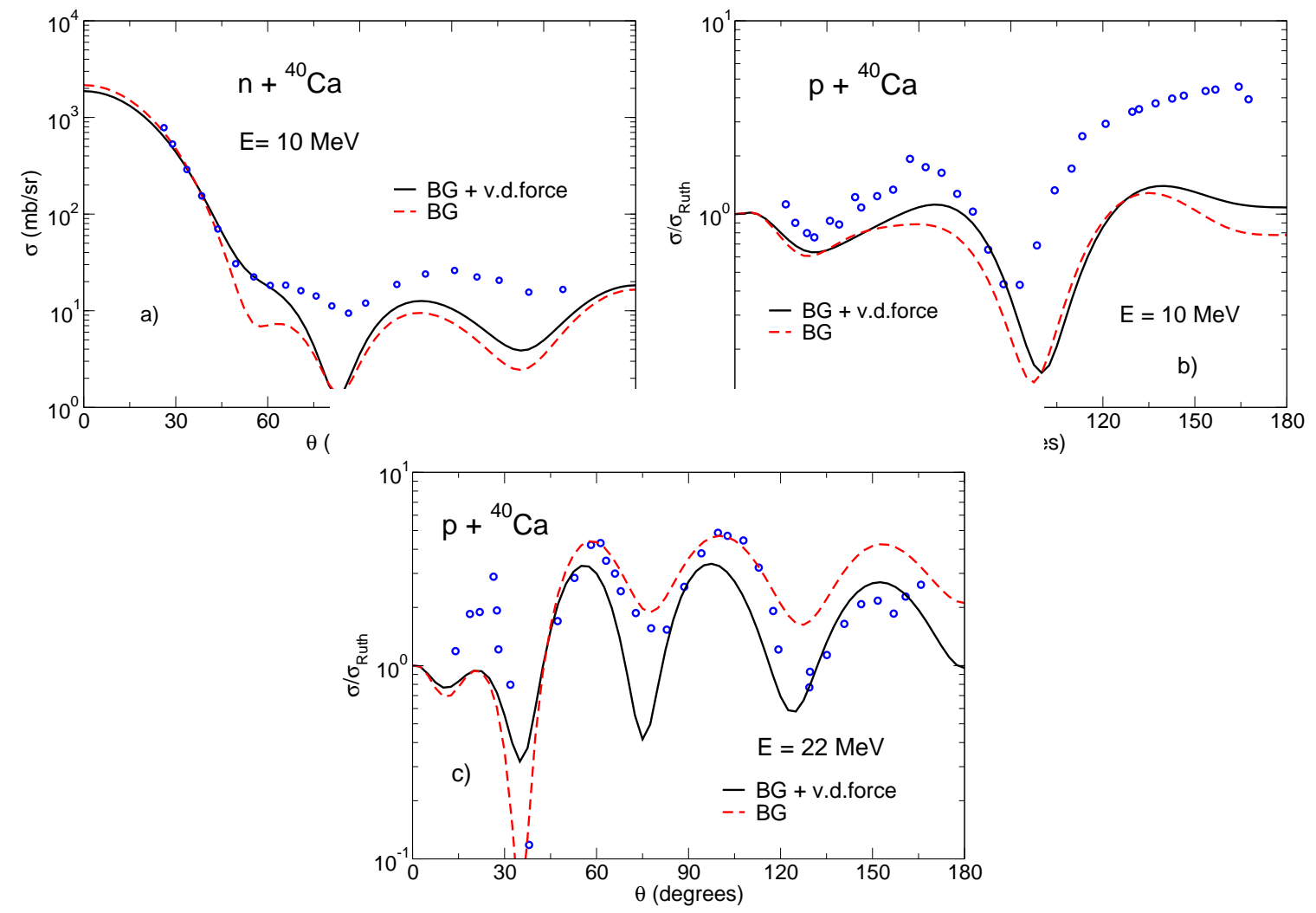

Figure 2. The neutron $(a)$ and proton $(b, c)$ scattering from ${ }^{40} \mathrm{Ca}$ at $E=10 \mathrm{MeV}$ $(a, b)$ and $22 \mathrm{MeV}(c)$ calculated using the Becchetti-Greenlees potential on its own (dashed lines) and with addition of velocity-dependent optical potentials (solid lines) in comparison with experimental data from [26, 27.

The adiabatic potential in the deuteron channel has been calculated in all three 
models, Ia, Ib and II, using the Hulthén deuteron wave function $\phi_{0}(r)$ [29]. The spinorbit potential both in the deuteron and proton channel were neglected. A proper treatment of the spin-orbit interaction in the ADWA requires calculating new tensor terms [30] for which no codes have been developed so far. Explicit calculations within distorted-wave-Born-approximation, performed here as a test for ${ }^{40} \mathrm{Ca}(d, p){ }^{41} \mathrm{Ca}$ at $E_{d}=10$ and $20 \mathrm{MeV}$, show that the change in the cross section at the first maxima (the region used to determine spectroscopic factors) due to neglecting spin-orbit interaction in the entrance and/or exit channels are smaller than $4 \%$. The effective adiabatic potentials $U_{d A}^{\text {eff }}$ are shown in Fig. 3. One can see that their imaginary parts are almost identical in all three models. The difference between the real parts of $U_{d A}^{\text {eff }}$, obtained in Ia and Ib, is very small. The model II real potential is noticeably different. To understand where the difference comes from, an approximation $r \gg R$ was made in all equation of section 4 , as is usually done in the Johnson-Soper model [31, together with the assumption of an infinite mass $A$. This approximations works well for all standard values of diffuseness and should work even better for $a_{\rho}=0.9 \mathrm{fm}$. It was found that in this approximation, the Ia and Ib potentials are just the sums of the effective nucleon optical potentials $U_{N A}^{\text {eff }}(R)$ while all the corrections due to these terms are very small. In the case of model II, the only significant contribution, that supplements the sum of nucleon optical potential $U_{n A}^{\mathrm{eff}}(R)+U_{p A}^{\mathrm{eff}}(R)$, comes from $B(R)$, which in the Johnson-Soper approximation reduces to $\rho(R) /(1-\rho(R))\left\langle T_{n p}\right\rangle_{V}$, where $\left\langle T_{n p}\right\rangle_{V}=\left\langle\phi_{0}\left|V_{n p} T_{n p}\right| \phi_{0}\right\rangle /\left\langle\phi_{0}\left|V_{n p}\right| \phi_{0}\right\rangle$ is the kinetic energy of the $n-p$ pair averaged over the short-range of their interaction. The same quantity is present in local-equivalent potentials in the nonlocal $(d, p)$ model [9]. It is strongly dependent on the choice of the deuteron model [15, 16], inducing an enhanced sensitivity of the $(d, p)$ cross sections to the high $n-p$ momenta, typical for short range $n-p$ separation.

To calculate the ${ }^{40} \mathrm{Ca}(d, p){ }^{41} \mathrm{Ca}$ cross sections, the overlap integral between ${ }^{41} \mathrm{Ca}$ and ${ }^{40} \mathrm{Ca}$ ground states is needed. It was taken from the nonlocal dispersive optical model (NLDOM) [32]. The updated parameters of this model are given in [14] where it was also shown that this overlap function can be approximated, with good accuracy, by the product of a single-particle wave function obtained for a Wood-Saxon potential well (with $r_{0}=1.252 \mathrm{fm}$, diffuseness $a=0.718 \mathrm{fm}$ and spin-orbit depth $V_{\text {s.o. }}=6.25 \mathrm{MeV}$ ) and the square root of the spectroscopic factor $S=0.73$. The same overlap function was used in all the calculations of this paper.

The deuteron distorted waves, generated by the ADWA potentials, were multiplied by the Perey factors of the corresponding models externally and then read back in by the TWOFNR code. The multiplication of deuteron distorted waves by the Perey factor reduces the $(d, p)$ cross sections by approximately $8 \%$ without noticeable changes in the shape of their angular distributions for all three models. The proton distorted wave in the exit channel were also multiplified by the nucleon Perey factor. This reduces the transfer cross sections further down by about $5 \%$. The ${ }^{40} \mathrm{Ca}+\mathrm{p}$ rather than ${ }^{41} \mathrm{Ca}+\mathrm{p}$ potential was used in the exit channel to remove the remnant term, as discussed in [33].

The Perey factors $P(R)$ are shown in Fig. 4. They are almost identical for the 
Three-body problem with velocity-dependent optical potentials: a case of $(d, p)$ reactions13

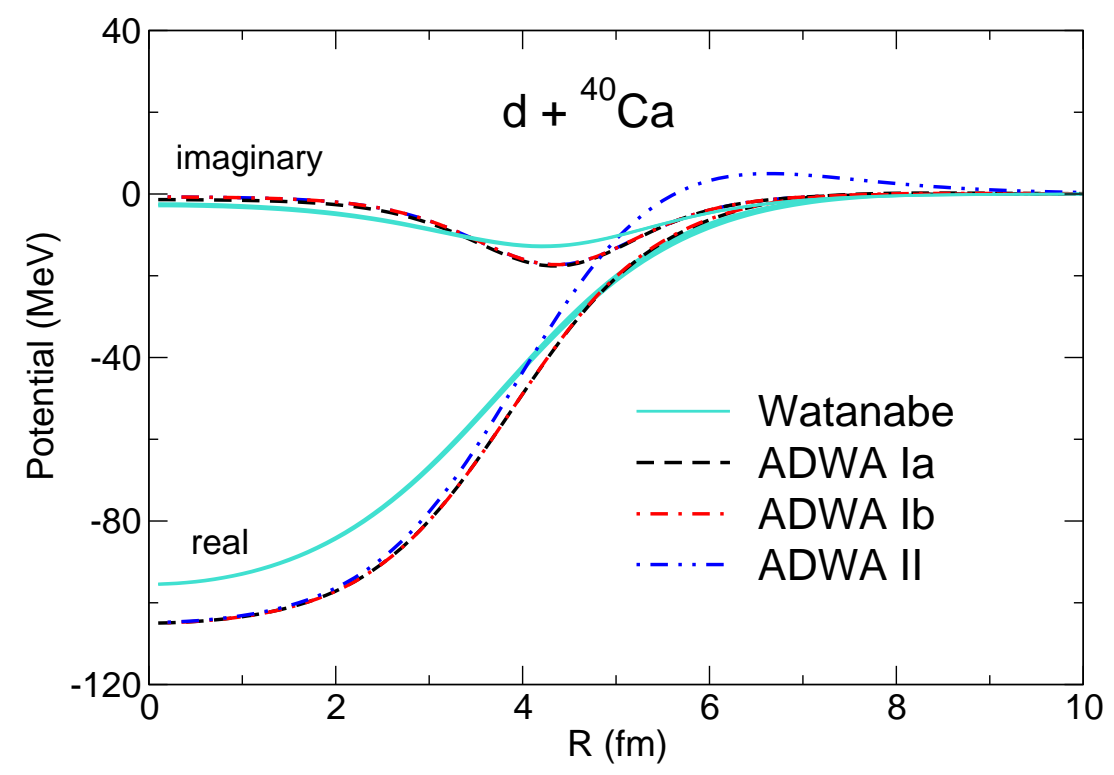

Figure 3. The adiabatic $d-{ }^{40} \mathrm{Ca}$ potentials obtained in models Ia, Ib and II (dashed, dot-dashed and dot-dot-dashed lines) in comparison to the $d-{ }^{40} \mathrm{Ca}$ potentials from the Watanabe model (thick band).

Ia and Ib models, being approximately equal to the square of the nucleon Perey factor $P_{N}$ from Eq. (9), also shown in this figure. The reason behind this near equality is the same as the one, discussed above, that causes the adiabatic Ia and Ib potentials to be very similar. Due to the short range nature of $\phi_{1}(r)$ the model Ib value of $S(R)$ is approximately equal to $\frac{1}{2}\left[\nabla_{R} F_{n}(R)+\nabla_{R} F_{p}(R)\right]$. Furthermore, if $F_{n}=F_{p} \equiv F$ then equation (48), of which $P(R)$ is the solution, in the limit of infinitely large mass $A$ transforms into $P^{\prime} / P=2 m / \hbar^{2} \nabla F$, where $m$ is the nucleon mass. This equation is the same as Eq. (6) that determines $P_{n}$ or $P_{p}$, apart from a factor of two, which causes the solution for $P(R)$ to be the square of $P_{N}$. The same $P(R)$ value is given by the model Ia: $P(R)=P_{n}(R) P_{p}(R) \approx P_{N}^{2}(R)$. This similarity gradually disappears with decreasing diffuseness of $\rho(r)$ because the validity of the Johnson-Soper $r \gg R$ estimate for $S(R)$ is getting lost. As for the Perey factor for model II, it comes from a differential equation with $\tilde{S}(R)$ that does not reduce to $S(R)$ and, therefore, it cannot be equal to the $P(R)$ from model I. Figure 4 shows that it is smaller.

The calculated ${ }^{40} \mathrm{Ca}(d, p){ }^{41} \mathrm{Ca}$ cross sections are shown in Fig. 5 5 a. One can see that models Ia and Ib give similar predictions for these cross sections, which are very close to those calculated in the Johnson-Tandy local model with the original BecchettiGreenlees potential. However, model II gives a significantly different result, both in shape and magnitude, which is the consequence of the additional term in Eq. (57), $B(R) /(1-\mathcal{R}(R))$, determined by high $n-p$ momenta in deuteron through $\left\langle T_{n p}\right\rangle_{V}$. It has been pointed out in [17] that nonlocal non-diagonal potentials, that couple the first Weinberg component $\chi_{0}$ to all the other ones, also strongly depend on the deuteron model choice through their different high $n-p$ momentum content and that a large 


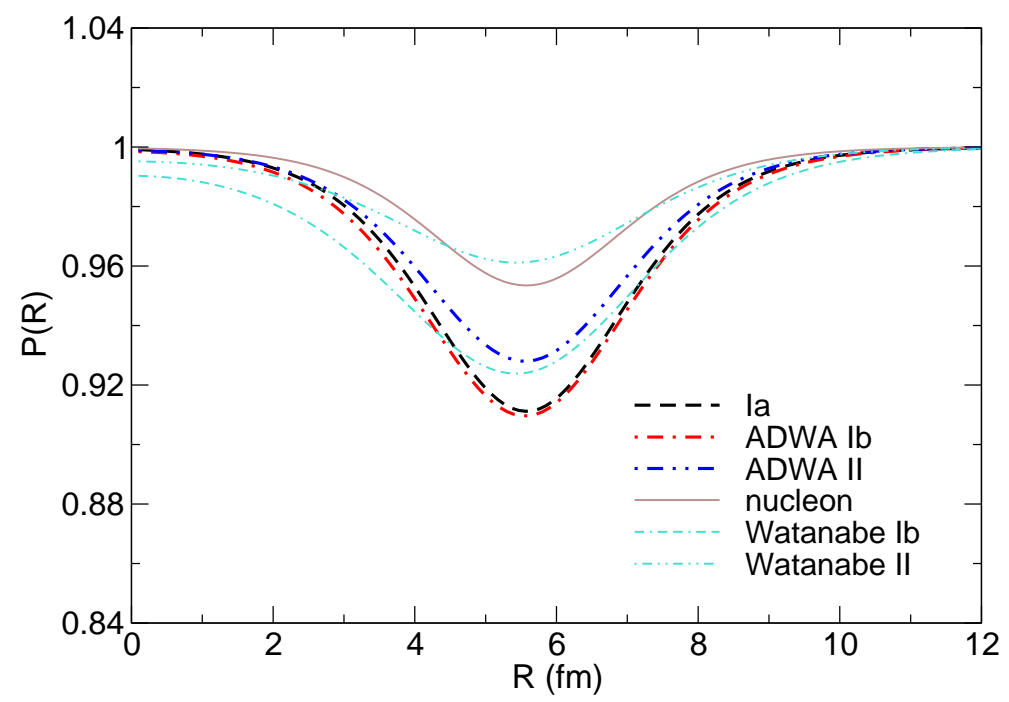

Figure 4. The Perey factors in the $d-{ }^{40} \mathrm{Ca}$ channel, calculated in the ADWA and the Watanabe models, in comparison with the $p-{ }^{40} \mathrm{Ca}$ Perey factor (thin line) given be Eq. (9). For the Ia model, the ADWA and Watanabe Perey factors are identical.

number of Weinberg states is needed to overcome this model-dependence. It was also shown in [17] that an alternative expansion of the three-body wave function $\Phi(\boldsymbol{R}, r)$ over the CDCC basis does not generate significant $n$ - $p$ momentum dependence, which has also been confirmed by rigorous Faddeev calculations in [18].

The first term of the CDCC expansion is given by the Watanabe folding model and for velocity-dependent nucleon optical potentials the Watanabe potentials can be obtained by replacing $\phi_{1}(\boldsymbol{r})$ with $\phi_{0}(\boldsymbol{r})$ everywhere in section 4 . The Watanabe potentials, obtained in models Ia, Ib and II, are very similar (see Fig. 3) because $B(R)$ is now determined by the average $n$ - $p$ kinetic energy over all the coordinate space, which is much lower than $\left\langle T_{n p}\right\rangle_{V}$. The Perey factors calculated in models Ib and II are smaller and wider. Their influence on $(d, p)$ sections is similar to the ADWA case. The Watanabe cross sections are shown in Fig. 5b. There is no significant difference between all three models. Some difference between the Watanabe cross sections obtained with and without velocity-dependent forces,using the original Becchetti-Greenlees potential only, is seen at very small angles.

The calculated ${ }^{40} \mathrm{Ca}(d, p){ }^{41} \mathrm{Ca}$ cross sections are compared in Figs. 5 a and $5 \mathrm{~b}$ to the experimental data from [34]. Both the ADWA and the Watanabe approaches predict $30 \%$ smaller cross sections as compared to the experimental data, thus suggesting that the spectroscopic factor should be larger than the NLDOM value of 0.73 used in these calculations. However, it was shown in [35] that using a different optical potential, such as local dispersive-model optical potential from [36], results in smaller spectroscopic factors. Also, a contribution from other reaction mechanisms could be responsible for disagreement between the predicted and measured cross sections. 

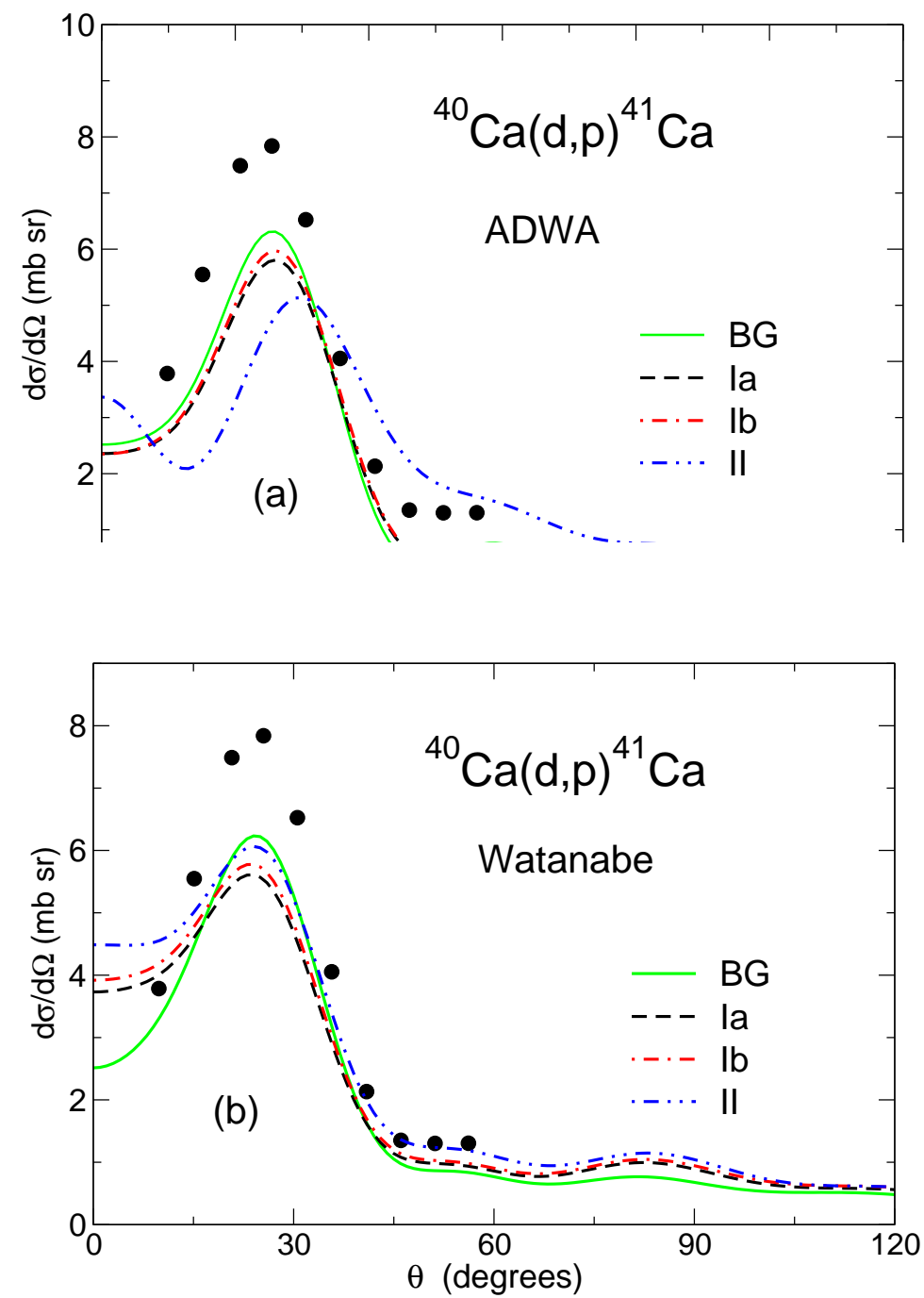

Figure 5. The ${ }^{40} \mathrm{Ca}(d, p){ }^{41} \mathrm{Ca}$ cross sections at $E_{d}=20 \mathrm{MeV}$ calculated using velocity-dependent optical nucleon potentials in three different models of the ADWA $(a)$ and Watanabe $(b)$ approach in comparison to the standard local calculations with Becchetti-Greenless optical potentials.

\section{Summary and conclusions}

In this paper, velocity-dependent optical potentials that have a form consistent with a spatially-variable mass of a nucleon interacting with nucleons of the target, have been considered within the context of the three-body $A+n+p$ problem. For such a class of potentials an exact local-equivalent two-body model exist. As a consequence, there are two ways to formulate the three-body problem and to write down the corresponding Schrödinger equation. The first one is based on nucleon potentials with only first derivatives over the $p-A$ and $n-A$ coordinates, while the second one is based on original velocity-dependent potentials that contain both first and second derivatives on these coordinates. There is no obvious connection between these two representations at a formal level. 
Three-body problem with velocity-dependent optical potentials: a case of $(d, p)$ reactions16

The main reason to look into the velocity-dependence of optical potentials was to study the manifestation of nonlocal effects in $(d, p)$ reactions. Velocity-dependence is a particular form of nonlocality that can be derived for a general nonlocal potential by considering only a linear on kinetic energy term in its Taylor expansion over a variable that determines the nonlocality range. A three-body Schrödinger equation with velocitydependent potentials could be an easier task to solve than the one that involves nonlocal potentials of a general nature. It has been solved in the adiabatic approximation, which accounts for deuteron breakup and provides the wave functions at small $n$ - $p$ separations, relevant to the $(d, p)$ problem. This results in distorted waves $\chi(\boldsymbol{R})$ determined from a two-body differential equation with an adiabatic potential that could be constructed in three different ways.

The local-equivalents of velocity-dependent potentials, originally proposed in [2, 4], have a strongly repulsive surface feature, originating due to a very small diffuseness of velocity-dependent term, which is impossible to understand. Increasing the diffuseness, as suggested in the present paper, removes such an unphysical behaviour. Application of the velocity-dependent force, proposed in this work, together with the local part fixed by Becchetti-Greenlees systematics, to the ${ }^{40} \mathrm{Ca}(d, p){ }^{41} \mathrm{Ca}$ reaction revealed a large difference between the calculations based on three-body Schrödinger equation with first derivatives only (model I) and the calculations that include both the first and the second derivatives (model II). This difference is easily explained by an additional term in model II associated with the high $n-p$ momenta in the $n-p$ kinetic energy in deuteron averaged over the short range of the $n-p$ interaction, similar to what has been observed in a more general nonlocal problem discussed in [15]. Based on recent studies in [17, 18] it is reasonable to expect that this difference will most likely decrease when the three-body Schrödinger equation is solved beyond the adiabatic approximation. Indeed, the $(d, p)$ calculations, performed here in the Watanabe model (which comes from the lowest term of the CDCC expansion), do not show any difference between models I and II.

The energy of the ${ }^{40} \mathrm{Ca}(d, p){ }^{41} \mathrm{Ca}$ reaction has been chosen here because of the availability of experimental data both for the reaction and for the elastic scattering in the entrance and exit channels. However, comparison between the theory and experiment suggests that the spectroscopic factor of 0.73 , rooted in the NLDOM (a theory that forges a link between the nuclear structure and nuclear reactions [32]) and used in the calculations, is small. This contradicts a previous study at a lower deuteron energy [14] with the same overlap integral and could be explained by a need for a different $p-{ }^{40} \mathrm{Ca}$ optical potential. It is also possible that the reaction mechanism at this energy is not sufficiently understood. More theoretical work as well as more ${ }^{40} \mathrm{Ca}(d, p){ }^{41} \mathrm{Ca}$ reaction measurements around $20 \mathrm{MeV}$ are needed to understand the spectroscopic factor of ${ }^{41} \mathrm{Ca}$ as determined from transfer reactions.

Finally, to make a judgement about the importance of a velocity-dependent force in $(d, p)$ reactions, this force should be, first of all, unambiguously extracted from an independent source. Fitting this force to elastic scattering data will not provide a unique solution. Fixing this force from nuclear structure calculations while fitting the local part 
Three-body problem with velocity-dependent optical potentials: a case of $(d, p)$ reactions 17

of the optical potential only could be an optimal way to go forward. More research is needed in this direction. A more realistic treatment of optical potentials will help to reduce the uncertainties of spectroscopic information extracted from $(d, p)$ reactions.

\section{Acknowledgements}

This work was supported by the United Kingdom Science and Technology Facilities Council (STFC) under Grants No. ST/L005743/1 and ST/P005314/1.

\section{References}

[1] Jaghoub M I, Hassan M F and Rawitscher G H 2011 Phys. Rev. C 84, 034618

[2] Jaghoub M I 2012 Phys. Rev. C 85024606

[3] Jaghoub M I and Rawitscher G H 2012 Nucl. Phys. A 87759

[4] Zureikat R A and Jaghoub M I 2013 Nucl. Phys. A 916183

[5] Ghabar J N and Jaghoub M I 2015 Phys. Rev. C 91064308

[6] Green A M 1962 Nucl. Phys. 33218

[7] Perey F and Buck B 1962 Nucl. Phys. 32353

[8] Deltuva A 2009 Phys. Rev. C 79021602

[9] Timofeyuk N K and Johnson R C 2013 Phys. Rev. Lett. 110, 112501

[10] Timofeyuk N K and Johnson R C 2013 Phys. Rev. C 87064610

[11] Titus L J and Nunes F M 2014 Phys. Rev. C 89034609

[12] Rosset A et al 2015 Phys. Rev. C 92044607

[13] Titus L J, Nunes F M and Potel G 2016 Phys. Rev. C 93014604

[14] Waldecker S J and Timofeyuk N K 2016 Phys. Rev. C 94034609

[15] Bailey G W, Timofeyuk N K and Tostevin J A 2016 Phys. Rev. Lett. 117162502

[16] Bailey G W, Timofeyuk N K and Tostevin J A 2017 Phys. Rev. C 95024603

[17] Gómez-Ramos M and Timofeyuk N K 2018 Phys. Rev. C 98011601

[18] Deltuva A 2018 Phys. Rev. C 98021603

[19] Li W, Potel G and Nunes F M 2018 Phys. Rev. C 98044621

[20] Johnson R C and Tandy P C 1974 Nucl. Phys. A 23556

[21] Nagels M M, Rijken T A and de Swart J J 1978 Phys. Rev. D 17768

[22] Satchler G R 1983 Direct Nuclear Reactions (New York: Oxford University Press)

[23] Tostevin J A University of Surrey version of the code TWOFNR (of M. Toyama, M. Igarashi and N.Kishida), http://www.nucleartheory.net/NPG/code.htm

[24] Johnson R C and Soper P J R 1972 Nucl. Phys. A 182, 619

[25] Becchetti F D and Greenless G W 1969 Phys. Rev. 1821190

[26] Tornow W et al 1982 Nucl. Phys. A 385373

[27] Dicello J F et al 1971 Phys. Rev. C 41130

[28] Saito K, Tsushima K and Thomas A W 1997 Phys. Rev. C 552637

[29] Hulthén L and Sugawara M 1957 Handbuch der Physik (Springer)

[30] Johnson R C 2015 Phys. Rev. C 91, 054604

[31] Johnson R C and Soper P J R 1970 Phys. Rev. C 1976

[32] Mahzoon M H et al 2014 Phys. Rev. Lett. 112162503

[33] Timofeyuk N K and Johnson R C 1999 Phys. Rev. C 591545

[34] Eckle F J et al 1990 Nucl. Phys. A 506159

[35] Nguyen N B et al 2011 Phys. Rev. C 84044611

[36] Mueller J M et al 2011 Phys. Rev. C 83064605 